\title{
CONSTRUCTION DU SAVOIR EXPÉRIENTIEL DES MALADES, ET RAPPORT AUX SAVOIRS DES ADULTES NON SCIENTIFIQUES
}

\author{
Olivier Las Vergnas ${ }^{1}$
}

Les savoirs en santé ne sont plus exclusivement construits dans les processus de clinique organisés par le corps médical mais peuvent résulter aussi de formes d'auto-clinique gérées par des groupes de malades, comme c'est le cas en rhumatologie, diabète, SIDA et pour des troubles psychiques ou des syndromes méconnus ou mal reconnus. La reconnaissance de ces savoirs expérientiels constitue une nouvelle étape de l'éducation thérapeutique avec laquelle les malades dépassent leur situation d'allégeance au classique pouvoir médical, pour assumer un rôle de production de savoirs, en position d'empowerment. Voilà qui renvoie à la question de la capacité que l'on peut s'accorder ou non de s'approprier -voire de produire- du savoir pertinent par rapport aux savoirs savants académiques. De fait l'enseignement formel initial étant calibré pour que 3/4 des élèves quittent les enseignements scientifiques avant le baccalauréat, la grande majorité en s'étant vu infligées des notes marquant une

1 Cité de la Santé - Universcience et Université de Paris-Ouest-Nanterre-CREF-AFA (EA 1589)

Recherches en communication, $\mathrm{n}^{\circ} 32$ (2009). 
incapacité à poursuivre, les cours de sciences doivent générer fréquemment un sentiment d'inefficacité personnelle durable. Or, la gestion d'une maladie chronique constitue un des rares cas d'appropriation autodirigée de savoirs scientifiques où des profanes peuvent être conduits à dépasser ce sentiment. Elle s'inscrit en cela dans les quelques pratiques qualifiables d'auto-science existantes, où un intérêt personnel peut conduire à construire du savoir savant personnel, en transgression des catégorisations scolaires et des stéréotypes qu'elles véhiculent.

Cet article s'appuie sur un travail de description des rapports des adultes aux savoirs savants. A partir d'une revue de la littérature scientifique et des textes internes aux organisations impliquées dans l'action culturelle ou éducative, ce travail, conduit actuellement par l'auteur, vise à déterminer les obstacles et les facilitateurs au développement de manifestations d'intérêt scientifique chez les adultes non catégorisés comme scientifiques lors de l'enseignement secondaire. Il cherche à décrire l'effet de cette catégorisation pour mieux comprendre les limites des politiques de développement de la «culture scientifique et technique » pour tous qui se révèlent en effet peu efficaces selon leurs promoteurs (Chavot et Masseran, 2010 ; Felt, 2010).

En effet, de nombreux travaux (Jurdant, 1973 ; Jacobi et Schiele, 1988) ont montré que les pratiques de vulgarisation ou d'action culturelle scientifique sont loin de réduire les écarts entre scientifiques et non scientifiques dans la société. A contrario, on constate qu'elles ne parviennent pas à transmettre massivement des savoirs et que paradoxalement elles consolident la représentation d'un clivage entre la minorité de la population considérée lors des études secondaires comme aptes à être scientifiques et ceux qui s'en sont révélés inaptes. Le renforcement de ce clivage au lieu de le réduire est sans doute la principale explication à la répétition systématique de discours politiques velléitaires s'inquiétant d'une « désaffection des sciences » dans la société (Schiele, 2005 ; Las Vergnas 2007).

A contrario de la vulgarisation étymologiquement descendante, certaines situations de constructions de savoirs savants par des non scientifiques pourraient être considérées comme des formes de transgression de cette déclaration scolaire d'inaptitude aux sciences, sous réserve qu'elles soient fortement motivées et autodirigées pour permettre aux personnes de dépasser cette prophétie scolaire d'incapacité. 


\section{Des patients observants aux malades experts}

Or, justement, une revue de littérature ${ }^{1}$ (Jouet, Flora et Las Vergnas, 2010) montre que pour les pathologies chroniques, des expertises propres aux malades émergent dans de multiples cadres comme sources reconnues de savoirs autodirigés. Aux stratégies classiques d'éducation thérapeutique descendantes, proposées par les soignants pour améliorer l'observance des traitements (d'Ivernois et Gagnayre, 2008 ; Simon et al. 2009), s'ajoutent de nouveaux courants issus des communautés de malades qui s'intéressent à la dimension formative des maladies (Aujoulat, 2007). Historiquement constitutive de l'idée de démocratie sanitaire, la reconnaissance de tels savoirs expérientiels acquis par les patients est l'une des caractéristiques de la période actuelle, comme en témoigne l'expression « Patient expert » apparue à la fin des années 80 (Lorig et al. 1985).

Une preuve du fait que les malades commencent à être reconnus comme capables de s'approprier des savoirs au point de devenir des experts, est qu'ils occupent des rôles d'interlocuteurs représentatifs. Ainsi, en janvier 2009, le colloque « Participation des usagers dans les établissements de santé : des principes aux expériences, des expériences aux principes » (tenu à la Cité de la santé de La Villette) a confirmé la généralisation de cette reconnaissance, sous des formes allant de la présence institutionnalisée d'une personne en charge des relations avec les usagers (82\%), à la participation massive de représentants des usagers aux conseils d'administration des établissements publics de santé, ainsi qu'à une place prépondérante des associations au comité des usagers.

Que ce soit au travers des associations autour du Sida (TRT5), des hépatites virales (CHV) ou des fédérations de maladies orphelines (AFM-FMO) $)^{2}$, et des associations membres du CISS, les usagers et patients sont maintenant présents dans les organismes de recherches

1 Ce premier paragraphe contient quelques emprunts à la note de synthèse "Construction et Reconnaissance des savoirs expérientiels ». in Pratique de formation : Analyses, $N^{\circ} 57 / 58$, Saint Denis Université Paris VIII, 2010 qui vient d'être consacrée par E. Jouet, G.Flora et l'auteur à cette question.

2 AFM : Association française contre les Myopathies ; FMO : Fédération française des maladies orphelines; L'AFM (Avec le téléthon) et la FMO (dans une moindre mesure) sont d'importants contributeurs de la recherche et à ce titre font également des choix d'orientation en la matière. Ces associations sont règlementairement encadrées pour ce faire. 
au travers de diverses commissions autant dans les institutions (OMS, établissements de santé, Leem ${ }^{1}$, Fondations, Mutuelles) ou les ONG et autres autorités indépendantes (Epstein, 1996 ; Rabeharisoa et Callon, 1999 ; Barbot, 2002). Au fil du temps, ces échanges se sont ainsi étoffés grâce à la législation (Jouet, Flora, Las Vergnas, 2010). Les associations se sont tout d'abord intéressées aux recherches sur les médicaments et les traitements pour privilégier dans un second temps les aspects de la vie sociale, personnelle et professionnelle des patients, en développant notamment des recherches-actions ou en s'y associant étroitement comme c'est le cas dans plusieurs pays d'Europe pour la recherche action en santé mentale Emilia (voir l'article de Jouet dans ce numéro).

\section{Deux directions pour l'acquisition des savoirs}

La littérature montre que si l'expérience de la maladie s'est installée comme une expertise reconnue, c'est que ces dernières décennies ont été marquées par trois courants d'idées complémentaires portés par trois différentes familles d'acteurs. D'une part, du côté des soignants, ont été développées les stratégies d'éducation thérapeutique avec pour but de donner aux patients la possibilité d'interpréter leurs symptômes et d'améliorer leur observance et leurs conduites (d'Ivernois et Gagnayre, 2008). D'autre part, du côté des malades, se sont constitués des mouvements communautaires visant à l'entraide, l'empowerment (Freire, 1964) et la reconnaissance de leurs spécificités (Barbot, 2002). Enfin, d'un troisième côté, celui des spécialistes en sciences de l'éducation', les thématiques de l'autoformation et des formations par l'expérience ont occupé le devant de la scène (Carré, Moisan et Poisson, 2010). Cette concomitance de courants a entraîné bien plus qu'une simple transformation de la capacité des malades à performer leur rôle de «bons patients », tel qu'il leur était prescrit par les soignants. D'un point de vue symétrique, elle a aussi fait émerger les moyens de favoriser l'expression et la valorisation des savoirs que le vécu de leurs maladies leur permettait acquérir.

Ainsi, en plus de la forme classique de l'éducation thérapeutique des patients (ETP), historiquement organisée par des soignants, est

1 Leem : Les entreprises du médicament http://www.leem.org/medicament/accueil. htm, [dernière consultation le 17/11/09].

2 L'auteur est chercheur invité dans une équipe consacrée à l'apprenance et aux formations autodirigées d'adultes. 
devenue visible cette aptitude des malades à construire par eux-mêmes des savoirs pertinents pour leur traitement à partir de leur propre expérience. La première est dirigée par les soignants alors que la seconde est autodirigée et codirigée par le patient ou des groupes de patients (Jouet, Flora et Las Vergnas, 2010). Toutes les deux visent à l'acquisition par les patients de savoirs, puis de connaissances, voire de compétences issues d'apprentissages informels qui s'ancrent dans l'expérience quotidienne de la maladie. Cependant, elles diffèrent par la nature de ceux qui en assurent la guidance et renvoient donc explicitement aux questions de la « direction » des apprentissages (Carré, 2005).

D'ailleurs, comme toutes les productions de savoirs « experts » à partir du quotidien profane, cette construction de savoirs émancipateurs ne va pas sans déclencher d'interrogations comme par exemple celles de Reach (2009) qui s'étonne que l'on puisse prétendre comparer des savoirs universitaires des médecins à ceux, trop empiriques des patients confirmant les résultats de l'étude de Stubbelfield et Mutha (2002) qui montrait qu'interrogés quant aux rôles dévolus aux patients, les soignants se réfèrent de façon majoritaire au modèle médical paternaliste et non au modèle de partage de décision.

De fait, en matière de savoirs des malades, la part entre savoirs académiques acquis par des éducations thérapeutiques descendantes et savoirs construits grâce à une auto clinique est directement dépendante du niveau de maturité des corpus de savoirs académiques dans la pathologie concernée. Elle semble déterminée par trois variables : (1) le volume et nature du corpus de savoirs médicaux déjà établis (2) les rôles des usagers dans la formalisation des savoirs expérientiels individuels (entraide, pairs aidants, scientificité des organisations), (3) les modalités d'un éventuel dialogue entre les patients et les tenants d'une éducation thérapeutique classique (Simon et al, 2009). Dans le cas de pathologies dont l'identification est émergente (comme certaines maladies orphelines), mais plus encore des pathologies très fréquentes mais longtemps sous-reconnues comme la fibromyalgie ou les syndromes de fatigue chronique, les savoirs expérientiels des patients constituent de fait la principale source actuelle de connaissance (Robert, 2007 ; HAS, 2010) au travers d'apprentissages à la fois autodéterminés, autodirigés et autorégulés. 


\section{La répétition des velléités de diffusion de la culture scientifique}

Replacée dans le contexte velléitaire des discours sur la culture scientifique pour tous, l'étude des appropriations de savoirs autodirigés par les malades permet une double mise en perspective. D'une part, une analyse des rapports des profanes aux sciences en général peut permettre de mieux comprendre la posture des malades vis-à-vis des savoirs académiques des médecins, des soins et des traitements ; d'autre part, symétriquement l'appropriation de savoirs par les malades peut fournir une étude de cas utile dans la lignée des travaux sur la vulgarisation (Jurdant, 1973 Jacobi, 1990), la socio-diffusion de la science (Jacobi, 1990), la communication scientifique publique (Fayard, 1988), la publicisation de la science (Paillard 2005) et les relations « sciences dans la société » (Chavot et Masseran, Felt, Bensaude-Vincent, 2010).

De fait, les efforts de partage des savoirs scientifiques qui se situent au cœur de ces champs n'ont pour l'instant pas obtenu de résultat notable au niveau de la société toute entière, comme le démontre leur répétition systématique. Tout se passe comme si des invariants sociétaux rendaient caducs ces efforts des politiques de diffusion de la culture scientifique. Déjà, Jurdant (1973) puis Boltanski et Maldidier (1977) suivi par Jacobi (1995) avaient montré que les publications de vulgarisation renforçaient la distance entre spécialistes et profanes. De même, aujourd'hui, plusieurs auteurs ont montré (Schiele, 2005, Felt 2010, Le Marec, 2010) que les discours ${ }^{1}$ des politiques sur la désaffection des sciences $^{2}$ ne faisaient que se répéter : depuis quatre décennies, adressés à la société toute entière, ils proposent toujours, sans jamais aboutir à un effet à grande échelle (Schiele, 2005), des plans d'action similaires visant à développer l'approche expérimentale des sciences à l'école, renforcer la place des sciences dans les programmes de TV et impliquer les citoyens dans les débats scientifiques. Une telle inefficacité des politiques sur des décennies peut sembler d'autant plus surprenante

1 Les analyses qui fondent l'avis $n^{\circ} 109$ « Communication d'informations scientifiques et médicales, et société : enjeux éthiques « du CCNE en ligne à http://www.ccneethique.fr/docs/CCNE-Avis_109.pdf constituent un exemple récent (10 février 2010) de ces discours.

2 Ces discours confondent aussi la question de la pénurie des inscriptions en premier cycle universitaire généraliste en sciences (dont nous ne traiterons pas ici) avec celle d'une nécessité de renforcer la culture et l'intérêt scientifique du plus grand nombre. 
que, dès lors que l'on se met à les chercher, on découvre çà et là des adultes de tout niveau et milieu qui se consacrent par intérêt à des activités de nature scientifique ${ }^{1}$. C'est le cas pour des personnes dénuées de formation scientifique initiale, mais devenues autodidactes dans un domaine qui les intéresse, comme les oiseaux, les cerfs-volants, 1'astronomie ; à ce constat déjà ancien (Bensaude-Vincent, 2010) se sont ajoutés des travaux (Astolfi, 1999 ; Giordan, 2008) qui montrent que les approches autodirigées de sujets scientifiques ou techniques permettent individuellement des appropriations efficaces de savoirs sans demander de prérequis scolaire, comme celles que Roux, Charvolin et Dumain (2009) recensent sous la dénomination de «passions cognitives ».

\section{La catégorisation scolaire comme modèle explicatif}

Ainsi, cette revue de littérature conduit à la question suivante : Pourquoi les politiques chantres du développement d'une "culture scientifique pour tous » ne peuvent- ils pas faire se multiplier largement de telles manifestations individuelles d'intérêt scientifique au lieu de répéter leur vaine déploration d'une présumée "désaffection des sciences » par le plus grand nombre ? D'où provient cet apparent paradoxe entre efficacité individuelle et échec sociétal ?

Un modèle explicatif peut être proposé en partant des déterminants forgeant l'image des sciences développée par les personnes non sélectionnées $^{2}$ comme technoscientifiques, qui constituent les trois quarts ${ }^{3}$ de chaque classe d'âge dans nos pays. Le système de formation scientifique initiale (en particulier au collège et au lycée) est tel que ces trois quarts ont été, dans leur grande majorité, écartés des sciences, par des mauvaises notes ; elles leur ont fait comprendre ${ }^{4}$ qu'ils n'étaient

1 Ce qui prouve par l'absurde s'il en était besoin qu'il n'y a pas d'impossibilité cognitive à ce que chacun puisse être passionné par tel ou tel aspect scientifique. Etre « non scientifique » n'est pas une fatalité biologique de ceux qui n'auraient pas la « bosse des maths ».

2 A savoir ceux pour qui l'enseignement des sciences a été arrêté avant le niveau du Baccalauréat.

3 Les $75 \%$ de non Bac S dans chaque classe d'âge actuelle.

4 Nous travaillons d'ailleurs actuellement à analyser l'effet à long terme des mauvaises notes. Nous postulons qu'elles conduisent à développer un «sentiment d'inefficacité personnelle » (SIEP), par analogie avec le « sentiment d'efficacité personnelle » (SEP), introduit par Albert Bandura qui empêcherait ensuite d'oser prétendre avoir 
pas autorisés à aller plus loin en termes de savoirs ou d'investigations scientifiques. Ainsi, dans ce modèle catégorisé, les adultes non scientifiques ne s'estiment plus capables d'auto diriger de leur propre chef des acquisitions de savoirs savants personnels. Ils ne peuvent donc qu'éventuellement en recevoir au travers du système d'enseignement ou de vulgarisation. A l'obstacle cognitif appelé épistémologique par Bachelard (1938), le souvenir de la catégorisation scolaire a ajouté un obstacle motivationnel (conatif) (Las Vergnas, travail en cours). Les rapports aux savoir abstraits sont alors limités à des circulations descendantes, appuyée sur les sens différents que prend généralement l'expression « savoirs scientifiques ». (1) En psychopédagogie, ce vocable est utilisé comme synonyme de " savoirs savants » ou « concepts scientifiques » pour désigner et analyser les connaissances abstraites qui se structurent dans l'intellect des individus par opposition aux savoirs quotidiens, dont ils sont séparés par l'obstacle épistémologique ${ }^{1}$. (2) En termes de dispositif scolaire, les savoirs scientifiques servent à définir les programmes et se déduisent de ceux créés et brassés par le monde de la recherche par des opérations qualifiées de «transpositions didactiques » qui les formatent pour l'enseignement descendant. (3) Quant à l'univers aujourd'hui mondialisé de la recherche scientifique, sa fonction même est décrite comme étant la production et la structuration des savoirs scientifiques. Le tableau T1 donne les caractéristiques de ces trois univers et des auteurs qui ont employé le vocable « savoirs scientifiques $»$.

partie liée de près ou de loin à quelque chose de scientifique, par un mécanisme de résignation apprise voire de prophétie auto réalisatrice..

1 Pour Vygotski, dans « pensée et langage », la traduction française de Françoise Sève utilise le vocable de « concepts scientifiques » opposés aux « concepts quotidiens ». Nous préférons quant à nous utiliser ici l'appellation « savoirs savants personnels » à celle de «savoirs scientifiques personnels » car faute de place nous ne traiterons pas ici en détail de la signification que nous pourrions donner à leur scientificité, c'est-à-dire à leur réfutabilité examinée à l'échelle individuelle. 


\begin{tabular}{|l|l|l|l|}
\hline Abr. & Dénomination & Mode de production & Principaux auteurs \\
\hline SSR & $\begin{array}{l}\text { Savoirs scientifiques de } \\
\text { la « recherche mon- } \\
\text { diale» }\end{array}$ & $\begin{array}{l}\text { Ils sont produits par } \\
\text { l'avancée des connais- } \\
\text { sances structurées par les } \\
\text { publications scientifiques } \\
\text { académiques }\end{array}$ & $\begin{array}{l}\text { Kuhn, } \\
\text { Popper, Latour } \\
\text { De la Solla Price }\end{array}$ \\
\hline SSS & $\begin{array}{l}\text { Savoirs scientifiques } \\
\text { scolaires (enseignés) }\end{array}$ & $\begin{array}{l}\text { Ils sont produits par une } \\
\text { «transposition pédago- } \\
\text { gique » qui consiste à } \\
\text { mettre les SSR « au for- } \\
\text { mat » de l'enseignement } \\
\text { formel descendant }\end{array}$ & $\begin{array}{l}\text { Verret, Chevallard, } \\
\text { Joshua }\end{array}$ \\
\hline SSP & $\begin{array}{l}\text { Savoirs savants per- } \\
\text { sonnels } \\
\text { (des non savants ou } \\
\text { enseignants) }\end{array}$ & $\begin{array}{l}\text { Ils résultent de l'assimila- } \\
\text { tion de SSS en lien avec } \\
\text { l'expérience personnelle } \\
\text { et de leur accommodation } \\
\text { aux représentations ou } \\
\text { conceptions préexistantes }\end{array}$ & $\begin{array}{l}\text { Bachelard, } \\
\text { Piaget, Vygotski. } \\
\text { Pour les adultes : } \\
\text { Malglaive, Bour- } \\
\text { geois }\end{array}$ \\
\hline
\end{tabular}

Tableau T1 : principaux univers sémantiques évoquant des « savoirs scientifiques »

Ainsi, pour les adultes non scientifiques, ces " savoirs scientifiques » sont en interaction selon des principes qui ne favorisent pas l'autodirection des apprentissages : les enseignements formels assurent dans le cadre scolaire la transformation des savoirs scolaires en savoirs personnels, les «transpositions didactiques " par lesquels les savoirs mis au point par les chercheurs deviennent des disciplines scolaires et s'organisent dans les programmes et les manuels, les vulgarisations directes par les chercheurs eux-mêmes ou médiatisée par des intermédiaires. A noter qu'il existe malgré tout un processus institué, s'appuyant sur la transformation des savoirs personnels, la « validation des acquis de l'expérience » $(\mathrm{VAE})^{1}$, mais que sa lourdeur le rend quasiment équivalent, malgré sa vocation initiale, à une reprise d'étude tardive.

Ce modèle descendant, respectueux des catégories scolaires, conduit aux processus représentés sur la figure 1 .

1 La VAE permet de transformer son expérience en diplôme grâce à un travail de formalisation montrant qu'elle a permis de maitriser les mêmes savoirs que ceux que l'on aurait acquis par la voie scolaire ; on peut la considérer comme un processus qui remonte des SSP vers les SSS. 


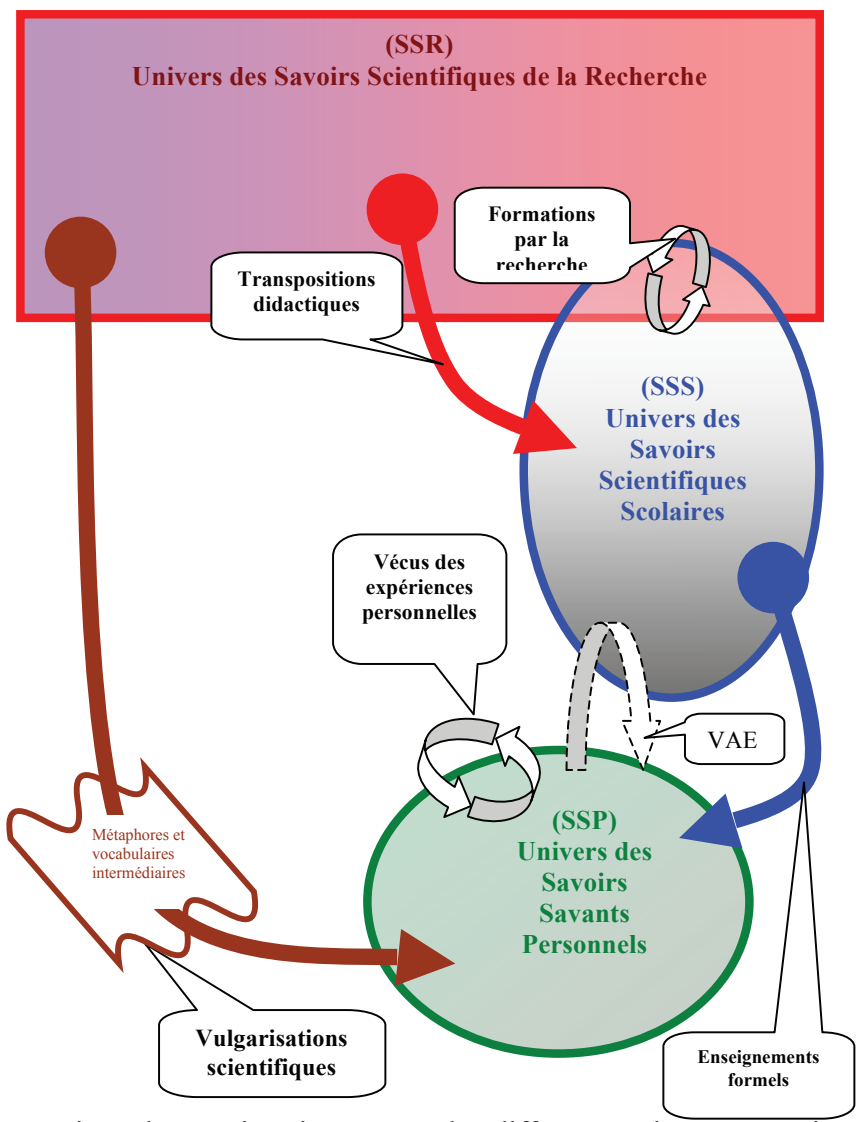

Figure 1 : interactions de premier niveau entre les différents univers sémantiques des « savoirs scientifiques » dans des disciplines scolaires (Las Vergnas, 2010 travail en cours)

\section{Acquisitions de savoirs en allégeance à la catégorie scolaire}

Dans ce modèle descendant, il n'existe que deux moyens pour les adultes non scientifiques de développer des savoirs savants personnels : profiter au mieux de la vulgarisation scientifique ou alors reprendre des études scientifiques. Plus généralement ces deux voies définissent deux options qui ne remettent pas en cause leur catégorie scolaire :

(1) la première (détaillée dans les trois premières colonnes du tableau T2) consiste à rester en retrait des « vrais scientifiques ", à leur faire allégeance, ce qui se montre par le développement d'un intérêt pour une version vulgarisée des savoirs scientifiques (lecture de vulga- 


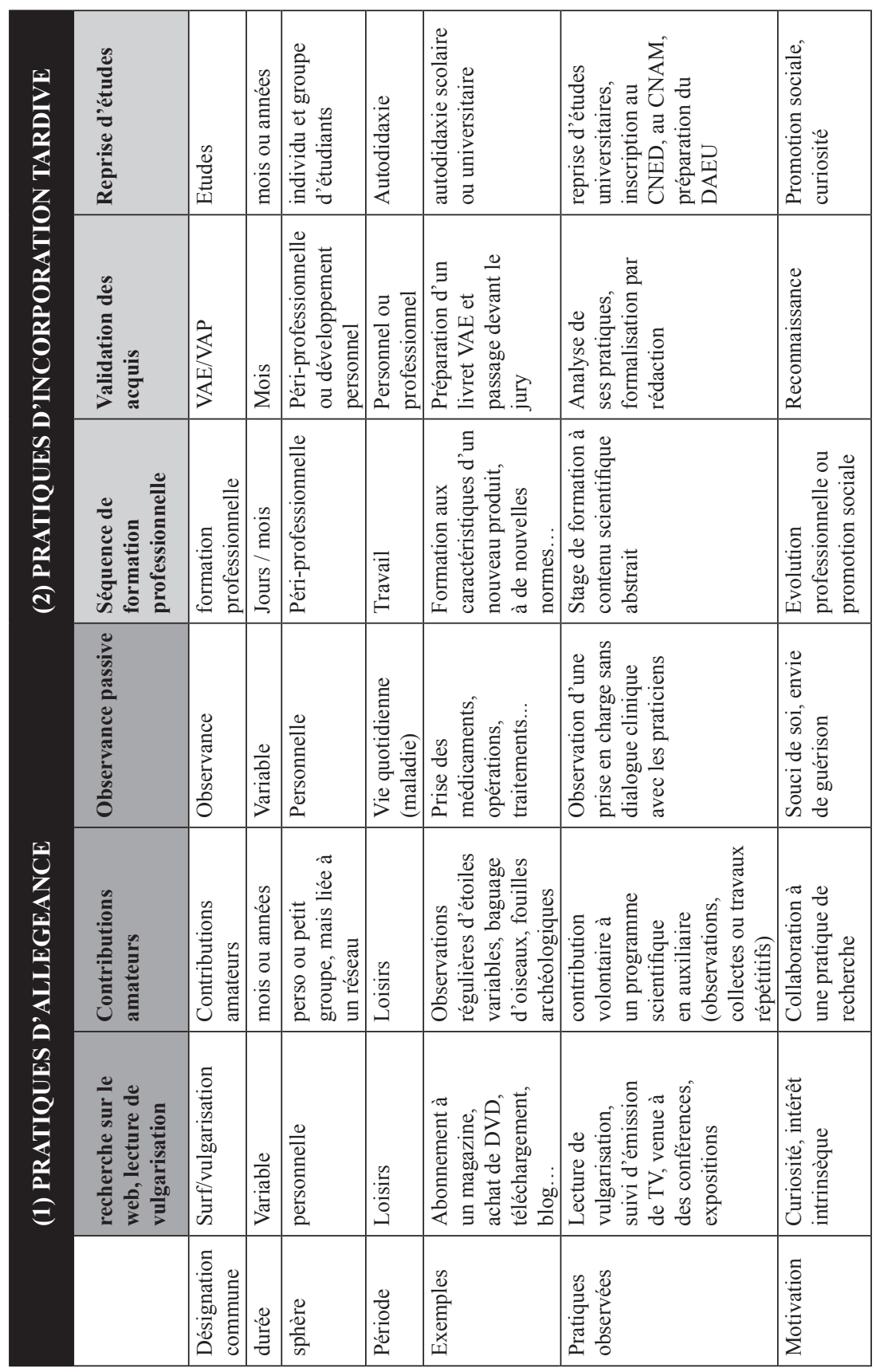

$\mathrm{T} 2$ : liste des acquisitions de savoirs en allégeance avec la catégorisation scolaire 
risation, contribution amateur à aider de vrais scientifiques dans un travail de routine, observance des prescriptions de son médecin).

(2) la seconde consiste se hisser a posteriori au sein de la catégorie des scientifiques, en s'inscrivant (tardivement donc) dans un travail de formation continue, de VAE ou de reprise d'études universitaire. Ces trois sous-cas sont figurés dans les 3 dernières sous-colonnes du tableau $\mathrm{T} 2$.

Concernant spécifiquement les savoirs malades, puisque nous avons introduit dans ce tableau "l'observance passive » d'un traitement, il est utile de compléter la figure 1 pour y introduire la relation avec les médecins traitants et soignants qui constituent une courroie de transmission des savoirs scientifiques issus de la recherche similaire au modèle scolaire ou de la vulgarisation (cf figure 2), en allégeance à la catégorie scolaire.

Les descriptions précédentes ne donnaient aucune place aux proches et aux groupes dans la construction des savoirs scientifiques. Or, l'analyse de la littérature sur le sujet, tant du côté des associations 


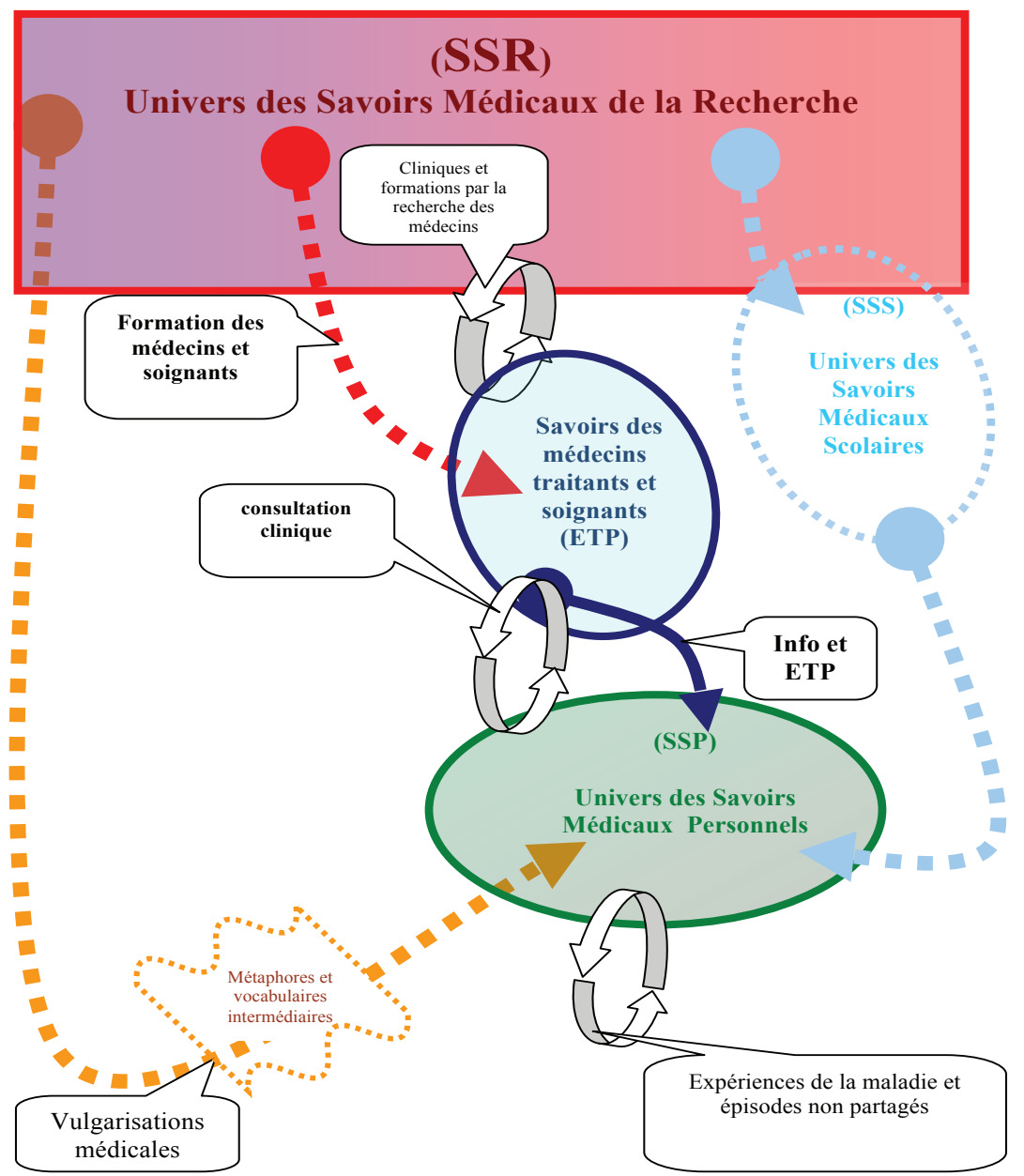

Figure 2 : positionnement de la chaine médicale (Las Vergnas, 2010 travail en cours)

de malades que des travaux en sciences de l'éducation montre que la parole et le travail collectif jouent des rôles clefs dans le repérage et les différentes phases d'assimilation de savoirs. Ils sont essentiels à des échafaudages de savoirs qui permettent la progression de chacun. Pour en tenir compte, sur la figure 3 , l'univers des interactions ${ }^{1}$ avec d'autres personnes a été ajouté à ceux de la figure 1 .

1 Il correspond aussi bien à expériences collectives ensuite individuellement théorisées ou a contrario vécues collectivement y compris au niveau de la métacognition. 


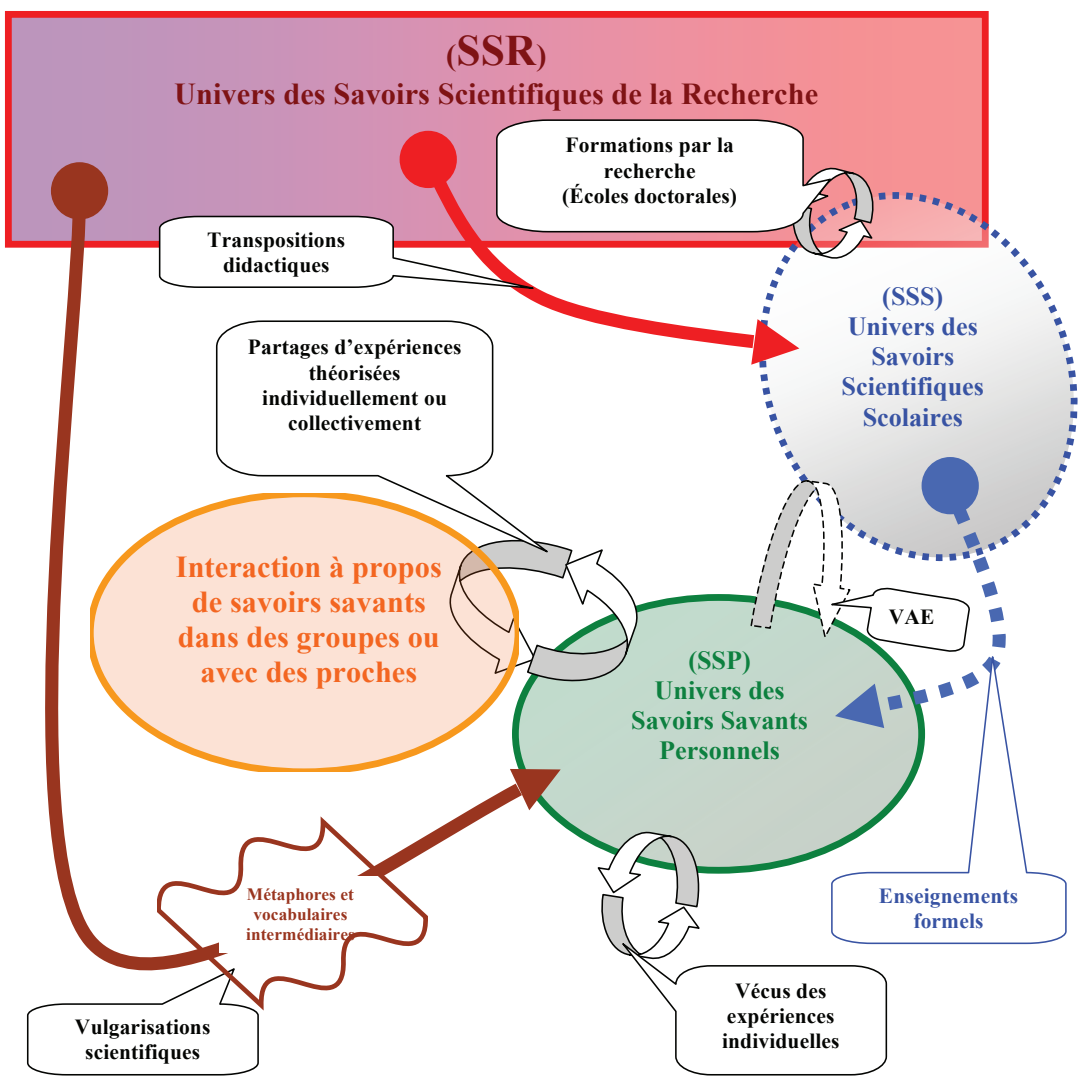

Figure 3 : positionnement de l'univers des proches ou d'un groupe (Las Vergnas, 2010 travail en cours)

Il est fait référence à des groupes que l'on pourrait qualifier d'« apprenants ", comme certaines associations, clubs scientifiques (astronomie, jeux de réflexion), techniques ou sportifs à forte dimension rationnelle (entrainement fractionné, sports mécaniques, vol à voile). Dans le cas de savoirs liés à la maladie, il correspond à l'univers des proches, aux groupes de pairs, comme les groupes d'entraide mutuelle (GEM) et des associations de malades. 


\section{Rapports aux savoirs transgressifs de la catégorisation scolaire}

En ayant introduit cette nouvelle dimension du groupe comme lieu de parole et d'assimilation, notre liste des manifestations d'intérêt scientifique (Tableau 2) peut être complétée. En effet, dès lors qu'un travail de métacognition peut être explicitement entrepris, ce qu'impose le dialogue avec les pairs, même les non scientifiques solaires peuvent se trouver impliqués dans de nouvelles pratiques, comme par exemple l'auto-clinique.

Même si celle-ci peut être individuelle, elle ne prend vraiment un sens de nouveau rapport au savoir que si elle s'accompagne de ce cadre de partage que constituent les proches ou une association de malades. Ainsi, les trois premières colonnes du tableau $\mathrm{T} 3$ présentent des appropriations volontaires de savoirs, dans une logique d'empowerment, s'appuyant sur des savoirs ou méthodes scientifiques non cantonnés aux disciplines scolaires ; il s'agit de l'auto-clinique, de l'investigation militante et du loisir scientifique à base de projets expérimentaux (Las Vergnas, 2007). Comme elles consistent non pas en réception de savoirs vulgarisés ou enseignés, mais en auto-production de nouveaux savoirs savants, la plupart du temps en s'appuyant sur cette dimension collective, nous les qualifions de « transgressions » de la catégorisation scolaire.

La quatrième colonne est là pour mémoire. Elle est consacrée aux recours au « raisonnement rationnel » qui renvoient à des conduites dans lesquelles est fait momentanément appel à la logique ou à un raisonnement hypothético-déductif au cours d'un tâtonnement pour résoudre un problème (abstrait ou concret), faire un choix entre plusieurs solutions ou mettre au point un dispositif. Ces épisodes sont diffus et souvent intériorisés, s'inscrivant dans un continuum de micro stratégies de tâtonnements ou de schèmes heuristiques dirigés par le besoin d'obtenir un résultat et non celui de s'approprier ou de mettre en œuvre des savoirs et prennent fin dès que le résultat attendu (réparation, bricolage, automédication, dosage, interprétation d'un symptôme ...) est atteint. Même si ces épisodes ne sont pas exactement " transgressifs " de la catégorie scolaire, on pourrait être tenté de les assimiler à des comportements transgenres intériorisés. 


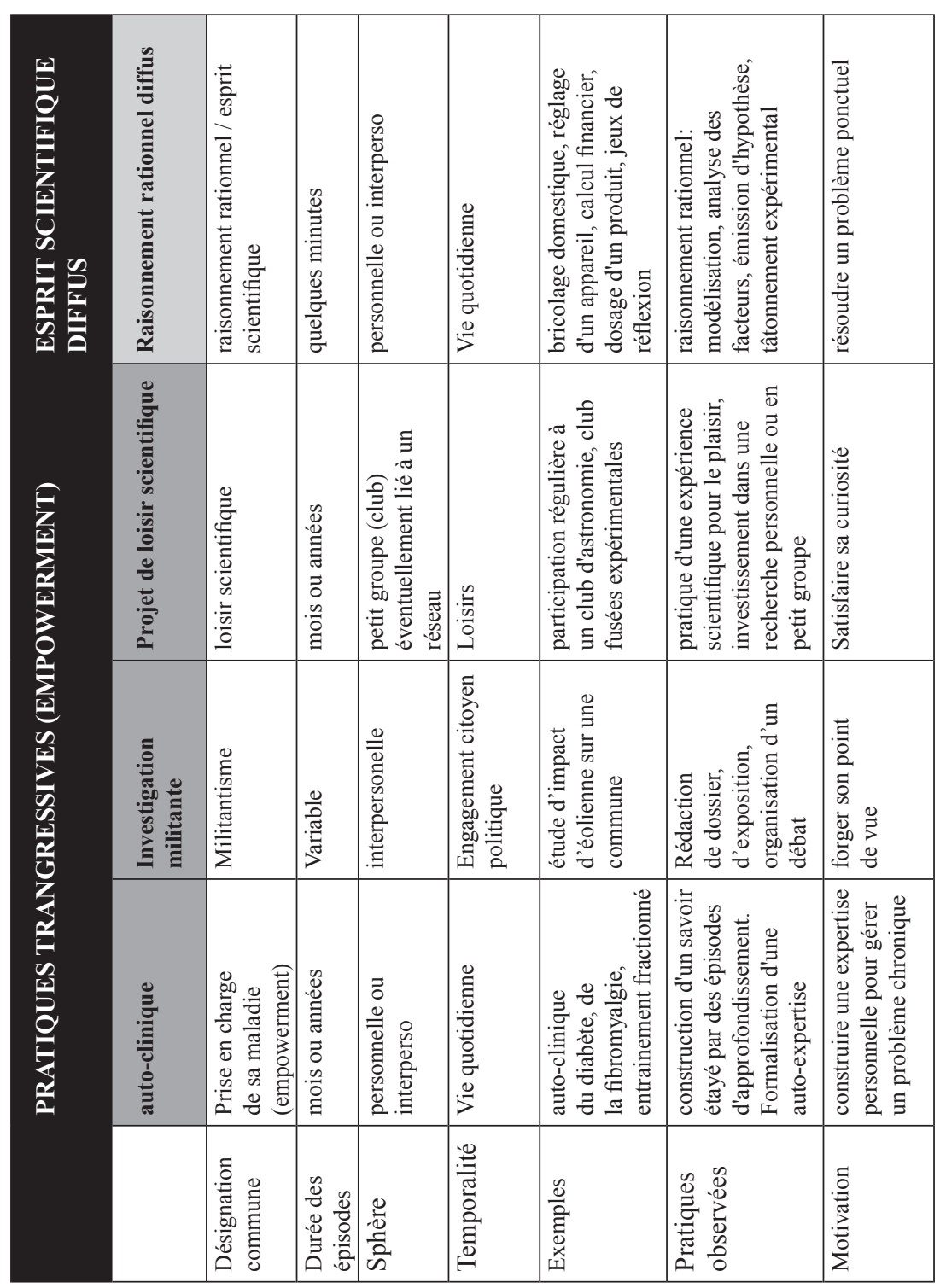

Tableau T3 : manifestations d'intérêt transgressives de la catégorisation scolaire

La gestion d'une maladie chronique dans une logique d'autoclinique peut donc ainsi constituer une des rares familles d'appropriation autodirigée de savoirs savants. Elle constitue donc l'un des trois cas (avec l'investigation militante et certaines formes de loisirs scienti- 
fiques expérimentaux) où des profanes peuvent être conduits à dépasser leur sentiment d'inefficacité vis-à-vis de la production de savoirs scientifiques et à s'atteler -individuellement ou en groupe- à un travail légitime de formation autodirigée à des savoirs scientifiques. En cela, la motivation à moins souffrir rejoint d'autres motivations à apprendre, allant de la curiosité pour un domaine de connaissance à des nécessités liées à la résolution d'un problème, à la pratique d'un sport, d'un loisir, d'une activité militante. Comme dans chacune de ces autres situations, la production de savoirs expérientiels étayés par les malades constitue une transgression de la catégorie scolaire.

La figure 4 en propose une schématisation appliquée à une maladie qui commence seulement à être prise en compte dans une clinique médicale (la fibromyalgie) et où les savoirs expérientiels sont centraux dans la création de tous les corpus de référence. Elle a été établie par l'auteur suite aux présentations des activités conduites dans le cadre d'associations de malades, lors de la douzième journée mondiale de la fibromyalgie, (12 mai 2010, Cité de la santé, Paris). 


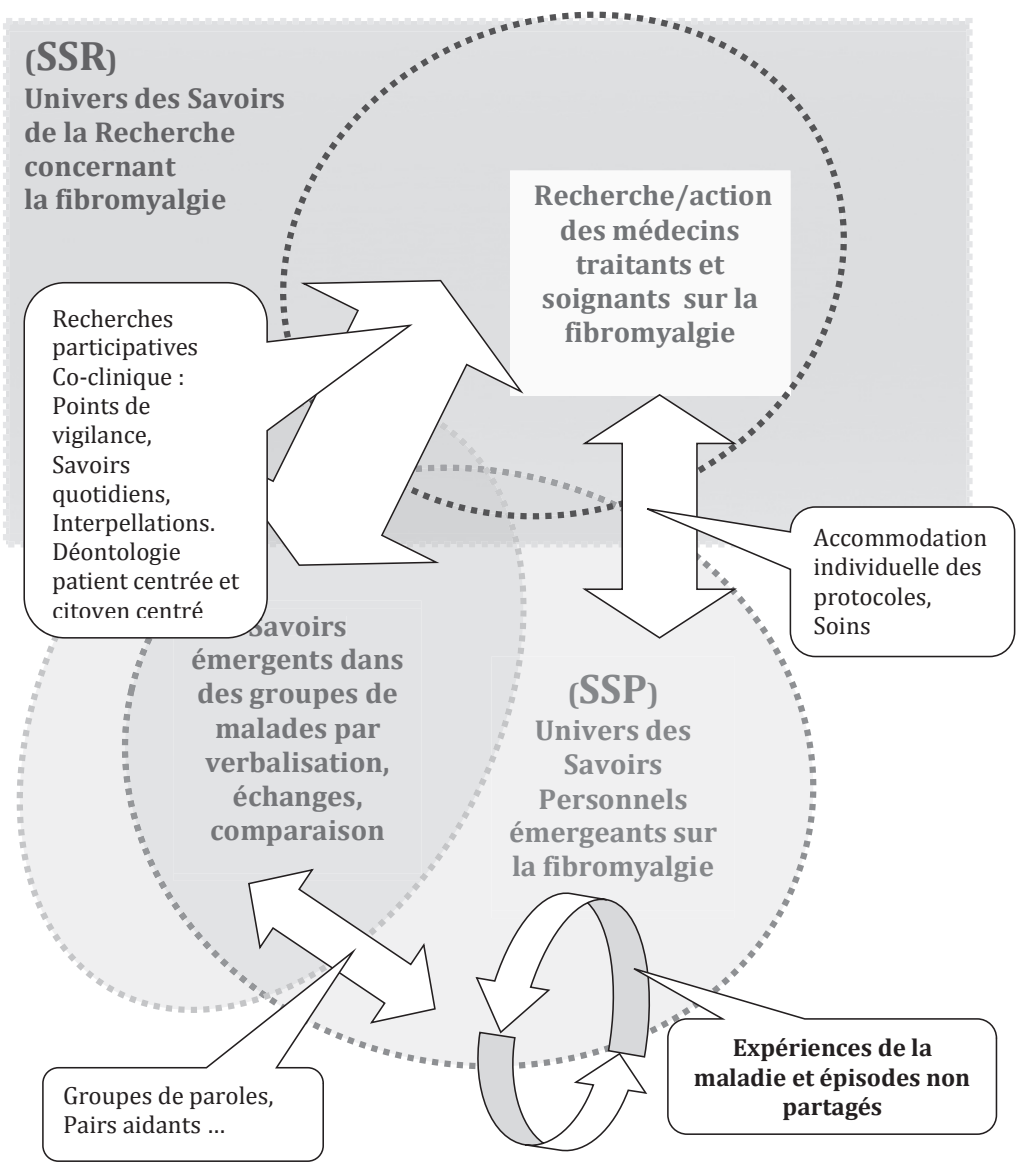

Figure 4 : interactions de savoirs concernant la fibromyalgie (Positionnement des activités évoquées lors du colloque du 12 mai 2010 à la cité de la santé)

\section{Discussion : sentiment d'efficacité et scientificité}

Nous avons formulé l'hypothèse que, dans la façon de penser des adultes non scientifiques, la mémoire de la catégorie scolaire cliverait les différents univers de savoirs et inhiberait les possibilités pour eux de considérer une connexion entre leurs raisonnements personnels et ceux des scientifiques. Pour eux, le recours à une application personnelle de la science pour résoudre un problème serait potentiellement 
vécu comme un comportement transgressif. Or, les malades engagés dans une auto-clinique semblent pouvoir, peut-être plus que d'autres au vu de l'importance de l'enjeu personnel, transgresser la limite des catégories scolaires surtout s'ils sont en interaction avec des groupes d'entraide mutuelle.

Voilà qui rejoint la question centrale de ce travail : quand les adultes s'autorisent-ils à de tels franchissements de la catégorie scolaire ? Est-ce que ces activités autodirigées peuvent contrecarrer d'éventuelles réminiscences des mauvaises notes ? Aident-elles à reconquérir un sentiment d'efficacité personnelle vis-à-vis des savoirs scientifiques ?

Une autre question clef est celle de la possibilité de qualifier la validité scientifique des savoirs acquis par des malades. Une grille d'exploration pourrait être bâtie selon plusieurs familles de critères (1) la nature des interactions et inter-relations avec des pairs et surtout avec l'univers scientifique ou médical (2) l'existence d'un cadre (par exemple dans un GEM, par l'observation collective de plusieurs cas similaires) d'analyse permettant de prendre du recul vis-à-vis de son seul cas personnel (3) de la capacité prédictive de ces savoirs et de leur réfutabilité (Popper, 1968).

Le travail présenté ici n'en est qu'au premier stade, celui de la proposition d'un modèle descriptif issu de revue de littérature et d'observations exploratoires en lien avec des groupes de malades. La phase suivante va consister à tester ce modèle à partir de données plus systématiques concernant des malades souffrant de troubles peu maitrisés par les soignants. Elle permettra ainsi d'explorer aussi ces questions de la scientificité et du sentiment d'efficacité personnelle. 


\section{Bibliographie}

Astolfi (1999). Les ateliers sciences et techniques, chemin vers l'abstraction et la réussite. Rapport d'étude. (dactylographié). Nanterre, CG92.

Aujoulat (2007). L'empowerment des patients atteint de maladies chroniques. Des processus multiples, auto-détermination, auto-efficacité, sécurité et cohérence identitaire, Thèse de médecine, Unité RESA, Louvain, Belgique.

Bandura (2003). Auto efficacité, le sentiment d'efficacité personnelle, Bruxelles, De Boeck.

Bachelard (1938). La formation de l'esprit scientifique, Paris, Vrin.

Barbot (2002). Les malades en mouvement, La médecine et la science à l'épreuve du SIDA. Paris, Balland.

Bensaude-Vincent (2010). Splendeur et décadence de la vulgarisation scientifique. In Chavot et Masseran (Eds). Dossier « les cultures des sciences en Europe », Question de communication, 17, PUN Nancy.

Boltansky et Maldidier (1977). La vulgarisation scientifique et son public. Une enquête sur « Science et vie ». Paris, Edition de l'EHESS.

Bourgeois et Nizet (1997). Apprentissage et formation des adultes, Paris, PUF.

Caria et Wils (2009). États des lieux des modes de participation des usagers dans les établissements de santé, présentation des résultats de l'enquête nationale, In Actes $d u$ colloque «Participation des usagers dans les établissements de santé, des principes aux expériences, des expériences aux principes », Paris, Cité de la santé/CSI.

Carré (2005). L'apprenance, vers un nouveau rapport au savoir, Paris, Dunod.

Carré, Moisan et Poisson (2010). L'autoformation, Paris, PUF.

Chavot et Masseran (Eds). (2010). Dossier « les cultures des sciences en Europe », Revue Question de communication, 17, PUN Nancy.

Chevallard et Joshua (1985), La transposition didactique, Du savoir savant au savoir enseigné, Grenoble, La Pensée sauvage.

Epstein (1996). Impure science, Berkeley, University of California Press.

Fayard (1988). La communication scientifique publique, Lyon, Chronique sociale.

Felt (2010). Vers la construction d'un public européen ? Continuité et rupture dans les discours politiques sur les cultures scientifiques et techniques. In Chavot et Masseran (Eds). Dossier « les cultures des sciences en Europe », Question de communication, 17, PUN Nancy.

Freire (1965). Educação como práctica da liberdadae [Education, pratique de la liberté]. Brasil, Paz e Terra.

Giordan et al. (2008). Savoirs Emergents, quels savoirs pour aujourd'hui ? Nice, éditions Ovadia.

HAS (2010). Fibromyalgie de l'adulte. Rapport d'orientation, Paris, Haute autorité de la santé.

d'Ivernois et Gagnayre (2008). Apprendre à éduquer le patient, Maloine, Paris.

Jacobi et Schiele (Ed.). (1988). Vulgariser la science, le procès de l'ignorance, Seyssel, Champ Vallon.

Jouet, Flora et Las Vergnas (2010). «Construction et Reconnaissance des savoirs expérientiels ». Note de synthèse du $\mathrm{N}^{\circ}$, Pratique de formation : Analyses, $\mathrm{N}^{\circ} 57 / 58$, Saint Denis, Université Paris 8.

Jurdant (1973, édition 2009). Les problèmes théoriques de la vulgarisation scientifique. ERSTU - Paris, édition des archives contemporaines. 
Kuhn (1983). La structure des révolutions scientifiques, [traduction française], Paris, Flammarion

Lahire (2004). La culture des individus, Paris, La découverte.

Lainé (2005). VAE, Quand l'expérience se fait savoir, Paris, Eres.

Las Vergnas (2007). La culture scientifique et technique comme idéologie, Alliages, 59.

Latour et Woolgar (1988). La vie de laboratoire, [tra. Française] Paris, La découverte.

Le Marec (2009). Chercheur de science. Préface in Jurdant. (édition 2009). Les problèmes théoriques de la vulgarisation scientifique. ERSTU - Paris, édition des archives contemporaines.

Lorig et al. (1985). " Outcomes of self-help education for patients with arthritis ». Arthritis and Rheumatism, 28 (6).

Malglaive (1990). Enseigner à des adultes, Paris, PUF.

Piaget et al. (1981-83). Le possible et le nécessaire, Paris, PUF.

Paillard (Ed.). (2005). La publicisation de la science, Grenoble, PUG.

Popper (1968). La logique de la découverte scientifique, Trad. française 1973, Paris, Payot.

Price (1963). Little science, big science, Colombia University Press.

Rabeharisoa et Callon (1999). Le pouvoir des malades. Paris, Presses de l'école des mines de Paris.

Reach (2009). «Une critique du concept de patient-éducateur » Médecine des maladies métaboliques, volume 3-1.

Robert (2007). Fibromyalgie, les malades veulent comprendre. Paris, Publibook.

Roux, Charvolin et Dumain (2009). Les « passions cognitives » ou la dimension rebelle du connaître en régime de passion, Revue d'anthropologie des connaissance, 3 (3).

Schiele (2005). Publiciser la science, pourquoi faire ? In Paillard, La publicisation de la science, Grenoble, PUG.

Simon et al. (2009). Education thérapeutique. Masson, Paris.

Stubbelfield \& Mutha (2002). Provider-patient roles in chronic disease management. Journal of Allied Health, 31.

Verret (1975). Le temps des études, Thèse, Université de Lille-III.

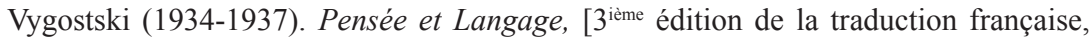
1998], Paris, La dispute. 